\title{
Research on public health crisis early warning system based on context awareness
}

\author{
Yingzhi $\mathrm{Li}^{\mathrm{a}}$ and Laiyuan $\mathrm{Xiao}^{\mathrm{b}, *}$ \\ ${ }^{\mathrm{a}}$ Division of Information Science and Technology, Wenhua University, Wuhan, Hubei, China \\ ${ }^{\mathrm{b}}$ School of Software, Huazhong University of Science and Technology, Wuhan, Hubei, China
}

\begin{abstract}
.
BACKGROUND: With the continuous expansion of urban scale and the increasing concentration of population, public health crisis has become an important part of urban residents' health management. The outbreak of the COVID-19 pandemic in Wuhan in 2020 has sounded the alarm.

OBJECTIVE: With the government at all levels to carry out the construction of urban Internet of things and information internet, the Internet backbone network has been built, deployed a large number of sensors, and collected a large number of urban situation data.

METHODS: In this paper, situational awareness technology is introduced into public health emergency services.

RESULTS: By constructing ontology, situational data and residents' health data are integrated. Through key technologies such as situational data collection, data fusion and data mining, real-time perception of environmental conditions of public health emergency scene is realized, and situational data fusion and situational information reasoning model are constructed.

CONCLUSIONS: The model is applied to the public health crisis emergency simulation system to verify the effectiveness of the model.
\end{abstract}

Keywords: Situational awareness, ontology technology, public health emergency

\section{Introduction}

The outbreak of the novel coronavirus epidemic in 2020 reminds us that national health is the foundation of all development. With the growth of urban scale and the increase of population density, public health emergencies have become an important factor seriously endangering people's physical and mental health and social stability [1]. However, as public health emergencies involve health data, gathering place situational information, medical information and her social data, human management is hard to realize. Therefore, how to integrate these complex multisource data for automatic public health crisis warning will become an important research topic [2].

Generally speaking, in 1994, Schilit et al. classified scenario information into three categories by means of enumeration:

- Computing scenarios - such as network availability, connection bandwidth, nearby devices, etc.;

- User scenarios - such as user configuration, location, people nearby, posture, behaviour, social relations, etc.;

\footnotetext{
${ }^{*}$ Corresponding author: Laiyuan Xiao, School of Software, Huazhong University of Science and Technology, Wuhan 430074, Hubei, China. Tel.: 13807180383; E-mail: xiao.1.y@ hust.edu.cn.
} 
- Physical scenarios - such as temperature, noise, light, traffic, etc.

Situational awareness is to fuse such situational information with the rest of the information, so that the computing device can generate corresponding response. The ultimate goal of context awareness is to enable the computer to actively retrieve information on the situation, further perceive the situation, and enrich the traditional human-computer interaction to provide better services. Context awareness technology should be able to complete realtime information collection integrating clear knowledge representation and semantics operation. Through information fusion technology, lowlevel scenario information is combined to generate highlevel information that can be identified by the application, and services are provided actively based on the information retrieved [3-5].

This paper constructs a public health crisis early warning system based on situational awareness. Its core concept is to collect the situational information of public places through sensors, fuse and analyse the situational information, realtime health data and network public opinion data, provide intelligent early warning services, and carry out targeted epidemic prevention and control.

This paper unfolds as follows. In Section 2, the model of collecting context information ontology, context information reasoning model and service configuration model are given. In Section 3, on the basis of expounding the key technologies of situational information, the public health crisis early warning system is designed. The Section 4 discusses the application of public health crisis early warning system in the case. At the same time, the influence of context information reasoning algorithm on early warning effect is analyzed. Finally, conclusions are drawn in Section 5.

\section{Key technologies}

\subsection{Ontology modelling fusion of context information collection}

The related information of public health emergency includes environment perception network data of crowded places, medical data of hospital information system, public health related network public opinion information, health bracelet and other body data information. These data include not only structured data of relational database, but also semistructured data of public opinion information and unstructured data of human temperature perception in crowded places According to the first mock exam, how to use these models to integrate these heterogeneous data becomes a key technical problem to be solved [6-8].

In the research, we propose to use the ontology based knowledge representation modeling method created by Gruber [9]. This method has two aspects.

- One is the unity of form, that is, different scene information uses the same representation, but this level is mainly to solve the problem of efficient storage and query of information [10].

- The second is the unified modeling on the semantic level. This system uses owl to describe ontology and offers seven steps to construct ontology [11]. Its goal is to obtain the knowledge of related fields, and to realize the common understanding of the knowledge of this field by determining the recognized vocabulary in this field, so as to keep it in accordance with the requirements of semantic interoperability in the Internet of things.

Our public health event entity can be represented by ontology class diagram and attribute relationship. According to the functional requirements, the common event ontology is divided into four categories in Fig. 1: location, data source, event type and event level, some of which contain subordinate subclasses. In this paper, the Resource Description Framework RDF triple < subject, predict, Object $>$ describes the local context information, residents' health data and public health macro data and their relationships, such 


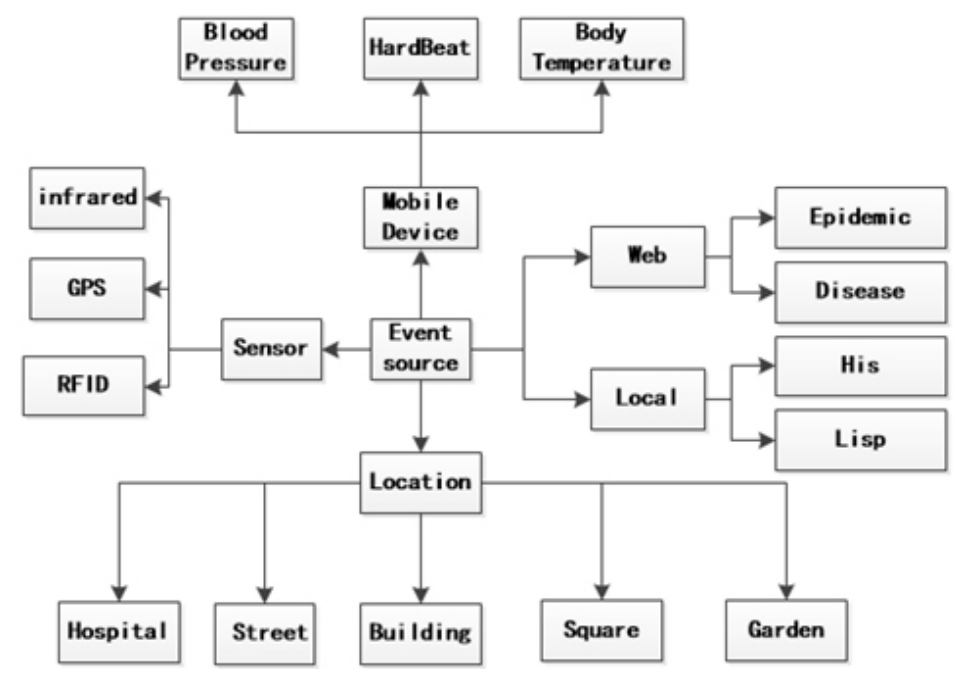

Fig. 1. Ontology class diagram.

as action path, measurement date, treatment information, measurement temperature, heart rate and other data, and forms local ontology networks such as local ontology of environmental data, local ontology of treatment data, local ontology of public opinion data, local ontology of residents' health, etc.

Subject and object are objects or individuals in ontology, while predicate is the attribute relationship defined in ontology. For example, in order to monitor the public place context model (member) of a specific area_ 05, locatedIn, grid_02), indicating that the current location of No. 05 personnel is No. 02 area; another example is (member)_05, temperature_36.7), indicating that the current monitoring temperature of No. 05 personnel is 36.7 degrees.

\subsection{Construction of the reasoning model of situational information}

Situational information needs to fuse the abovementioned local ontology of situational data, local ontology of medical data, local ontology of public opinion data, local ontology of residents' health and other molecular data through reasoning model to construct a global ontology to express the public health status information in a specific situation. Therefore, situational information reasoning is the key technology in the early warning system. The architecture is shown in Fig. 2.

\subsubsection{Situational reasoning algorithm}

This paper proposes a context-based reasoning algorithm based on semantic fusion, which mainly consists of two stages. The first stage is the semantic fusion connection stage based on attribute extension, which mainly realizes the concept connection of multiple local ontologies PI, and builds it into a unified knowledge base. The second stage is the information fusion stage based on Association calculation. This stage is mainly to calculate the distance of the association relationship between nodes in the generated local ontology network ontology $=(\mathrm{P}, \mathrm{e})$, so as to realize information fusion $[12,13]$. The main algorithms are as follows.

I. The first stage is a semantic fusion algorithm based on attribute extension: Input: ontology $=\{\mathrm{p}-\mathrm{o} 1, \mathrm{p}-\mathrm{o} 2, \mathrm{p}$-on $\}$ and the synonym-dict. 


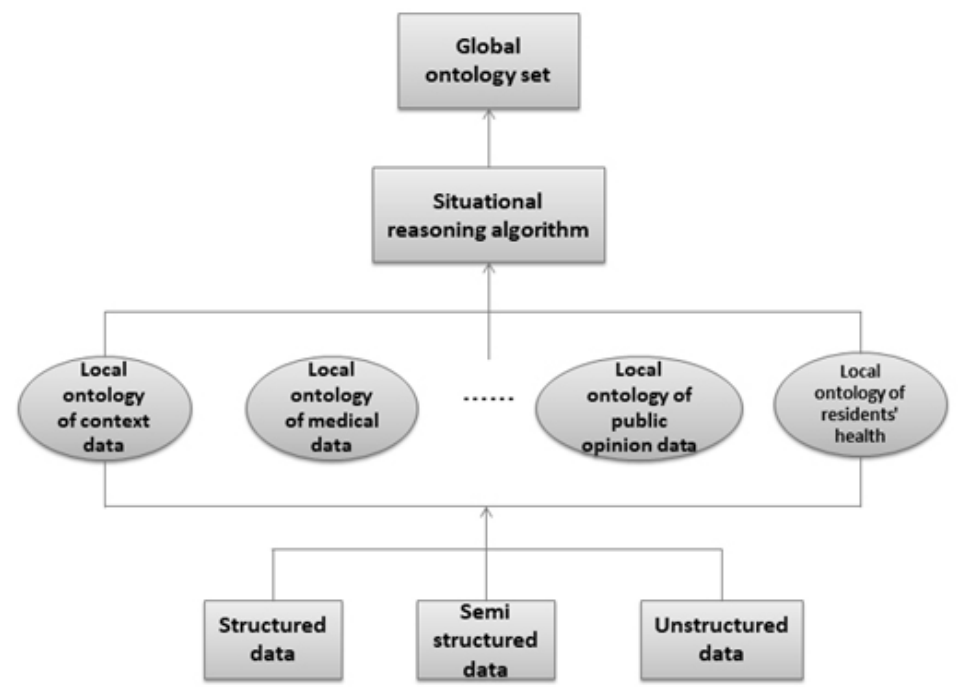

Fig. 2. Framework of context information reasoning system.

Among them: p-oi is the local ontology or combined context data formed by health data concept extraction; and dict is the association rule base constructed by the "islable" relationship defined by association rules.

Output: linked global ontology network g-ontology.

Step 1: loop for every P-Oi in P-Ontology

Step 2: loop for every Eij in P-Oj;

Step 3: if Eij in Dict;

The synonym of eij is extended to p-oj as a new attribute of eij;

end if

end loop

end loop

Step 4: loop for every two P-Oi and P-Oj

Step 5: if L- Oi and L- Oj have the same entity merge two entities

end if

end loop

In addition, when it is assumed that E1 and E2 can reach the entity set from two entity nodes, the calculation formula of eij is as follows.

$$
\text { cons }=\sum \mathrm{R} \mathrm{Ei} * \mathrm{R} \mathrm{Ej}
$$

II. The second stage is information fusion algorithm based on Association computing

Input: global ontology network graph g-ontology after synonym merging, $\mathrm{P}$ is the set of points, $\mathrm{E}$ is the set of edges.

Output: global ontology network graph g-ontology after heterogeneous concept fusion.

Step 1: loop for two entities

Step 2: if deep $<=3$

Step 3: calculate the correlation degree of the current node $\mathrm{Ri}+1=\mathrm{Ri}-1 /($ root of the length of the set $\mathrm{P}$ )

Step 4: if the current node has been traversed before 
Step 5: $\mathrm{R}=\operatorname{Max}(\mathrm{Ri})$; end if

Step 6: save the current node to Ei data set and En data set as well as the correlation

Step 7: depth traversal is associated with the current node end if

end loop

Step 8: for every entity in Ei and En

Step 9: if Eii same as Eni

Step 10: The correlation coefficient

cons $=\sum \mathrm{rEI} * \mathrm{r}$ en

end if

end loop

Step 11: if cons $>1$

Step 12: P-Oi and p-Oj are merged into one point;

else if $\mathrm{R}>2$

Step 13: Connect P-Oi, P-Oj, and add this edge to E

\subsubsection{Context based reasoning association service library}

Through the collection module, the system directly obtains the direct scene information such as the object location, crowd density, crowd temperature distribution, the current temperature and humidity of the object environment, and the medical treatment situation of the object location. By fusing the direct scene information of the above dimensions and the public opinion trend information, the system realizes online and offline information reasoning, and obtains the implied public health information of the object location. Then, it provides the basis for invoking the corresponding crisis and emergency response services.

The reasoning rules in this paper are designed based on health level 7, ISO/IEEE 11073 and IHE pcd-01 IR standards [14], which are the main standards for data sharing between sensor equipment and online information system. After obtaining the current situation through the above two steps of reasoning and description, we need to push the corresponding services according to the situation. The primary task of push service is to establish service classes for various kinds of information monitoring services in the process of public health event monitoring. Table 1 shows the major types of situational monitoring services.

\subsection{Generation of service configuration library}

The efficiency of reasoning engine is obtained through the information fusion of ontology fusion technology. The context information and business services are defined independently in the system, and the reasoning rules of services are transformed into the service configuration model in the Table 2.

The service configuration model is the core of the inference engine. The inference engine realizes the match between the situation and the service, which reduces the call control time and ensures the realtime service call. However, in the actual use process, in order to avoid the data distortion of local nodes caused by the failure of sensor equipment such as equipment aging and line delay [15], the system will also use the adjacent online and offline context to determine the necessity of large scale services such as broadcast services, so as to avoid any adverse consequences and unnecessary costs caused by this service. 
Table 1

Service list table

\begin{tabular}{ll}
\hline Service name & Description \\
\hline NWMS & Public opinion monitoring service \\
RTMS & Path monitoring service \\
PRMS & Personnel density monitoring service \\
ETMS & Environmental temperature monitoring service \\
PTMS & Human body temperature monitoring service \\
HIMS & Medical information monitoring service \\
HDMS & Health data real time monitoring service \\
DSMS & Equipment status monitoring service \\
BCS & Broadcasting services \\
CAS & Client notification service \\
EMS & Equipment management services \\
CLS & Component positioning service \\
SSS & Information push service \\
CES & Crisis assessment service \\
$\ldots$ & $\ldots$ \\
\hline
\end{tabular}

Table 2

Scene list table

\begin{tabular}{lllll}
\hline Situation scene & \multicolumn{4}{l}{ Public service regulations } \\
\hline ShCircle () & RTMS PRMS PTMS SSS CES \\
GdCircle () & RTMS EMS HDMS ETMS CAS \\
HisCircle & HDMS HIMS EMS CES BCS \\
NetCircle () & NWMS HDMS CES SSS \\
$\ldots$ & $\ldots$ & & & \\
\hline
\end{tabular}

\section{Public health crisis early warning system}

\subsection{Organizational structure}

The organizational structure of public health crisis early warning system is composed of context aware network, adaptation layer and data fusion center. The system uses distributed structure, deploys multilayer and multi perceptron in terminal network, and collects and stores perception data of summary level perception data, detail level perception data and professional perception data at different levels. This structure can ensure the flexibility and disaster tolerance of data. In addition, the security of data transmission and application is improved by using private network channel and authority control. Its organization chart is shown in the Fig. 3.

\subsubsection{Context aware network}

Its main task is to digitize the public health situation, collect the terminal detail data, and upload the situation data to the adaptation layer, so as to realize data fusion. In the real context aware network, there can be multiple terminal sensing terminals. These terminals can be divided on the grounds of different rules according to the characteristics of the environment. For example, the terminal perception center can be divided in terms of categories such as parks, networks, shopping malls and so on.

\subsubsection{Adaptation layer}

Its main task is to exchange data and information with the collected terminal data of different sources and structures. In this layer, middleware technology is used to build business gateway, resource gateway, data gateway and other logical gateways to collect data collected in context aware network. 


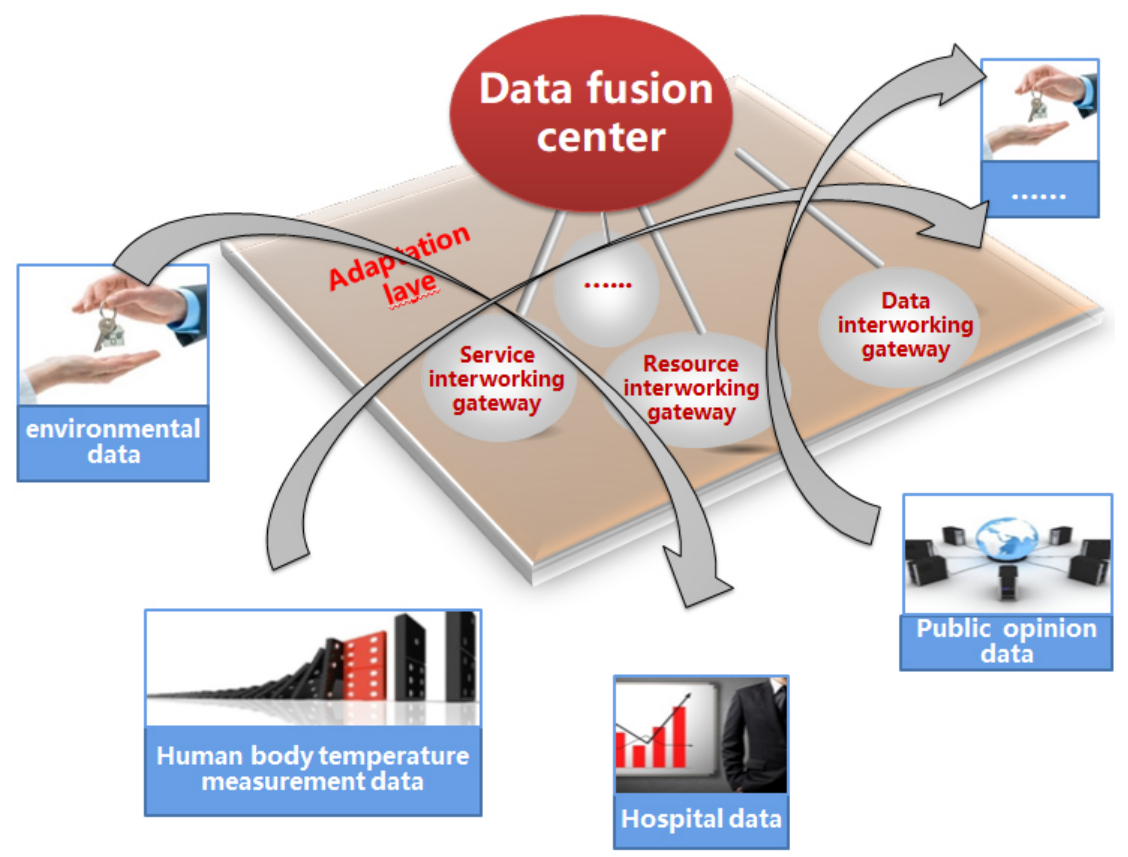

Fig. 3. Organization chart of public health crisis early warning system.

\subsubsection{Data fusion center}

Its main task is to build ontology model by transferring and connecting data of the adaptation layer. By fusing environment data, human body temperature measurement data, medical data and other terminal situation data, the summary information is obtained.

\subsection{System architecture}

The public health crisis early warning system is a technical system based on the perception framework of the Internet of things and multi-source ontology data fusion, and the collected data is reasoned by the engine. The structure of the public health crisis early warning system based on bus is shown in Fig. 4.

It can be seen from the figure that the public health crisis early warning system includes four parts: the acquisition layer the interface layer, the storage layer and the service layer.

\subsubsection{Acquisition layer}

This layer mainly obtains the main environment situation needed in the early warning system and the basic attributes of the gathering place, such as temperature, location, human body parameters, human body temperature, medical information, etc. through the hardware collector and software collector.

\subsubsection{Interface library}

This layer mainly calls real-time monitoring service and data extraction service through service registration component, obtains public health related information such as human health data, situation data and network data from sensors, medical treatment system, network public opinion and human health data collector, and calls mapping rule service and ontology construction service to generate local ontology model and integrate global ontology model. 


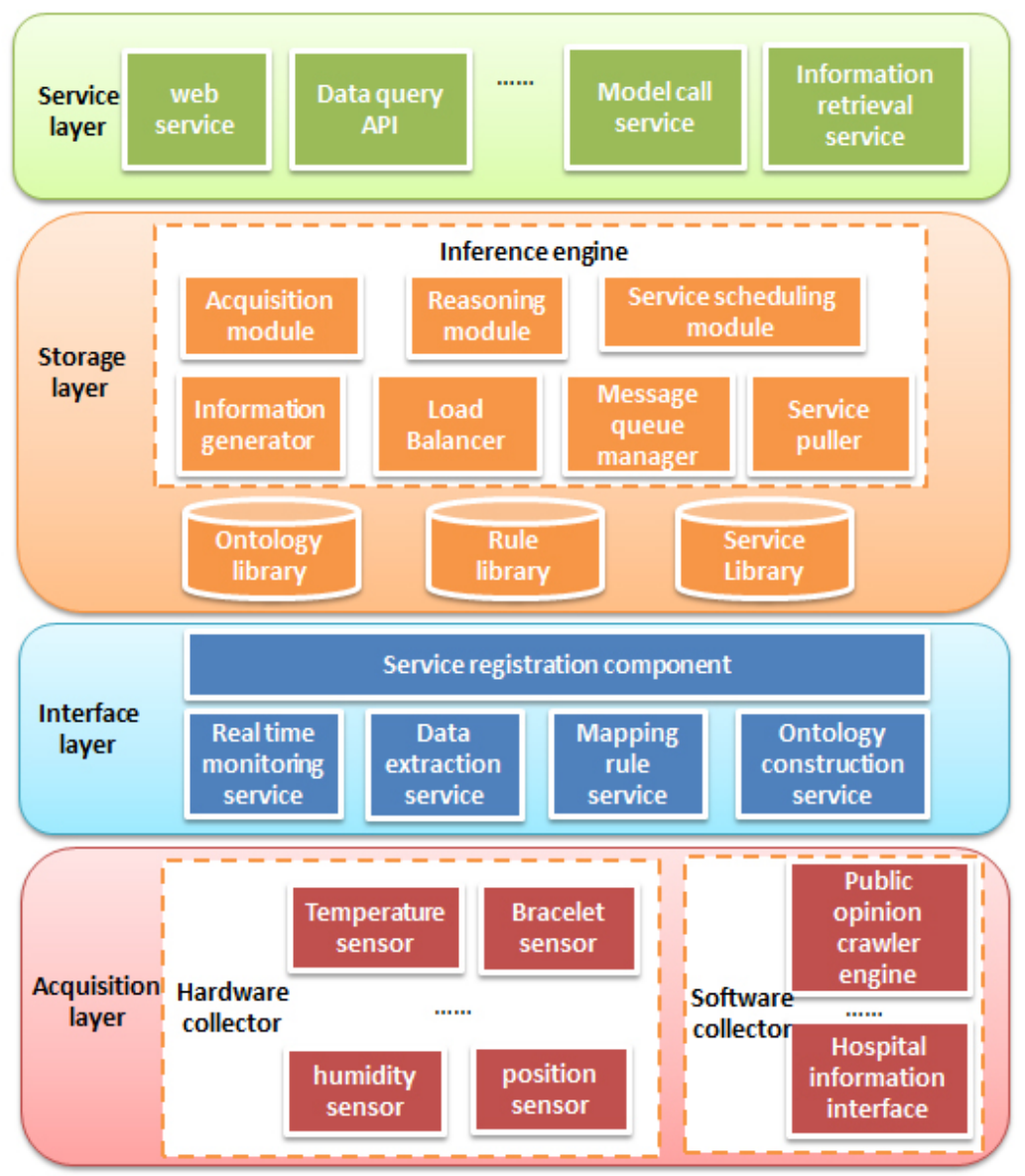

Fig. 4. Framework of public health crisis early warning system.

\subsubsection{Storage layer}

This layer is divided into two layers: data storage and reasoning engine. Data storage constructs a unified data using environment through data bus technology and completes the subsequent reasoning function through the corresponding configuration of context and service. This layer mainly includes ontology database, rule database and service database. These three kinds of independent storage databases ensure the independent expansion of context and service.

- The ontology library stores OWL Description patterns of various situations and reasoning rules of indirect situations [16];

- Rule base stores the corresponding rules of all kinds of situations and all kinds of business;

- The service library mainly stores all kinds of services that the system can provide;

Reasoning engine is a kind of middleware technology, which is used to analyze the situation of the acquired data in order to realize the intelligent fusion of public health information. Its components mainly include:

- Acquisition module: to realize the control of context awareness;

- Reasoning module: to realize the construction of fast operation situation;

- Service scheduling module: to ensure the efficiency of system operation; 
- Information generator: to realize the generation of comprehensive information;

- Load balancer: to ensure the stability of platform system operation;

- Message queue manager: to ensure the accuracy of platform operation;

- Business poller: to control the operating frequency of the sensor for control.

\subsubsection{Service layer}

This layer mainly provides the results generated by the context management engine to the end-user level of the system. The results of its reasoning engine are provided to the application system in the form of service.

- Main implementation methods: Web service, API, push service, search engine and other service methods [17];

- Main interaction mode: through mobile terminal, command screen, general browser, etc.;

- Main content: real time context information is transmitted to the proposed application through query interface service.

Above all, the public health crisis early warning system is an intelligent open system, which is centered on ontology data fusion and supplemented by software and hardware collectors to perceive the situation. It collects a variety of information metadata captured by various terminals, sensors and networks into the data center, and proposes the required early warning services according to the corresponding situation.

\subsection{Information flow}

The public health crisis early warning system is an intelligent open system, which takes the data fusion center as the core and multi-source context aware network as the antenna. It collects multi-source sensors, various public health related systems and a variety of information metadata captured in the network context into the adaptation layer, realizes data interconnection through the adaptation layer, and proposes the required services according to the corresponding situation. The specific operation process is shown in Fig. 5.

From Fig. 5, the system obtains the corresponding environmental data, human body measurement data, hospital data and public opinion data through the location information of the terminal node requested by context aware network.

After the context aware network collects data, it first mobilizes all kinds of running resources, cleans the collected data in the adaptation layer, then standardizes the data according to the rule base, and finally transmits multi-source data through the resource gateway, business gateway and data gateway, so as to complete the whole process of information processing.

After the completion of data processing, we can sort the data, classify and encode the data in the data fusion center, and build the information ontology database. According to the identified current situation, we can match the service configuration library, find out the corresponding service, and push it to the service terminal for automatic execution.

\section{Case analysis}

According to the research results of this paper, taking Jiang' an District of Hubei Province as a pilot, the early warning service based on scenario technology is integrated into the public health crisis emergency simulation system. As shown in Fig. 6, the system uses virtual simulation technology to create a unified 


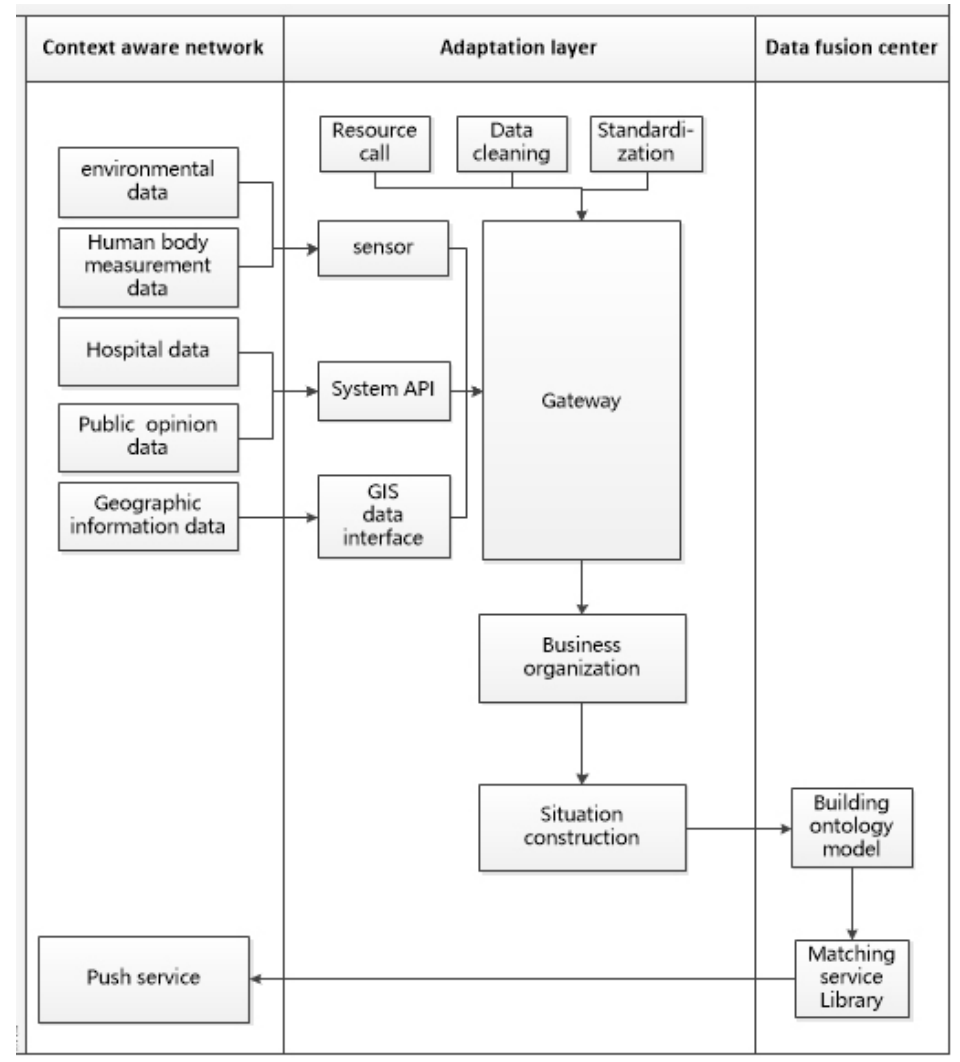

Fig. 5. Information flowchart of public health crisis early warning system.

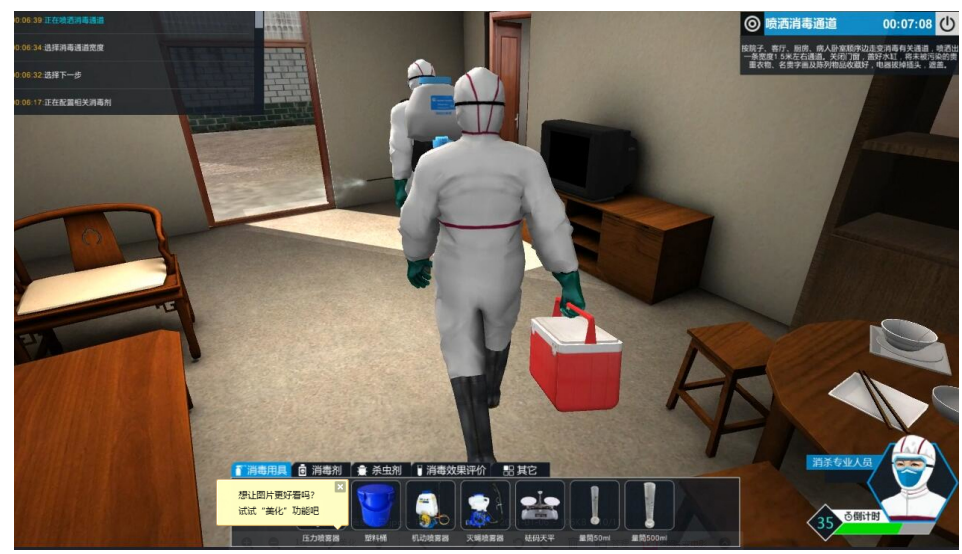

Fig. 6. Public health crisis emergency simulation system interface.

virtual environment of the entire system and integrates and controls a large number of entity models through the virtual environment. At the same time, the system also adds the context aware early warning model to provide realtime multi source public health data for the virtual environment. Through the combination of real data and virtual environment, the development process and standardized disposal 
procedures of public health crisis events were simulated, which provided an experiential training tool and environment for health emergency command and decision-making personnel, clinicians, public health professionals and so on.

At present, health departments at all levels continue to strengthen health emergency training exercises and have achieved remarkable results. However, there still exist many problems in grass-roots training, such as the lack of training teachers, nonstandard teaching materials, the lack of emphasis on professional content, limited training programs, and so on. Moreover, the training and actual combat drill requires exorbitant costs, which cannot achieve the expected effect. It is urgent to develop a combination of theoretical training and actual combat drill, which can stimulate the learning interest of health emergency personnel and comprehensively improve the quality of emergency response professional training system.

Since the pilot system was launched in Hubei Province, the accuracy of situational feedback has reached $90 \%$ through the application data of real scenarios, realizing the experiential learning method and stimulating the learning interest of health emergency personnel. The cost of professional training for emergency response level has been reduced by $30 \%$, and remarkable results have been achieved.

\section{Conclusion}

This paper proposes a public health crisis early warning system based on context aware technology and ontology fusion technology in order to fully perceive public places in the process of public health crisis emergency and explains in detail the key technologies such as context information ontology modeling fusion technology, context information reasoning technology, service configuration database technology and so on. Combined with the application case of public health crisis emergency simulation system, it is found that this study greatly reduces the risk of data management, lowers the costs of public health crisis response, and has high research and application value.

In the follow up work, we will improve each module of the system, and further study and optimize the breadth and depth of situational information. At the service level, the trend analysis and prediction of situational data will be intensively studied to provide more public health crisis early warning services.

\section{Acknowledgments}

This work was supported by the Natural Science Foundation of Hubei Province of China (No: 2020CFB675).

\section{Conflict of interest}

None to report.

\section{References}

[1] Wang H, Wen K. novel coronavirus pneumonia epidemic crisis in China. Accelerating China's Digital Transformation. 2020; 38(5): 393-395.

[2] Want R, Hopper A, Falco V, et al. The active badge location system. ACM Transactions on Information Systems. 1992; 58(10): 91-100.

[3] Gu T, Pung HK, Zhang DQ. A service- oriented middleware for building context awareness services. Elsevier Journal of Network and Computer Applications. 2015; 28(3): 1-18. 
[4] Guo C, Jiang L, Cai H. Automatic method of ontology constructingfrom relational database. Computer Engineering and Applications. 2012; 48(8): 115-120.

[5] Hazber MAG, Li R, Zhang Y, et al. An approach formapping relational database into ontology. Proc of the 12th Web Information System and Application Conference. 2015; 120-125.

[6] Li D, Yao Y, Shao Z. Concept supporting technology and application of smart city. Engineering Research. 2012; 88(12): 313-317.

[7] Bao Y, Sun H, Leng F, et al. User centered data cleaning process model in data warehouse environment. Computer Science. 2004; 31(4): 52-55.

[8] Noy NF, Mc Guinness DL. Ontology development 101: A guide to create your first ontology. http//protege.Stanford.edu/ publications/ontology/development/Ontology-101.pdf. 2012.

[9] Lee S, Do H. Comparison and analysis of ISO/IEEE 11073, IHEPCD-01 and HL7 FHIR messages for personal health devices. Healthcare Informatics Research. 2018; 24(7): 46-52.

[10] Li Y, Xiao L. Research on smart city service system based on context aware technology. Computer Engineering and Science. 2016; 38(3): 443-448.

[11] Dewan MAA, Granger E, Marcialis GL, Sabourin R, Roli F. Adaptive appearance model tracking for still-to-video face recognition. Pattern Recognition. 2016; 49(5): 129-151.

[12] Wang G, Zheng F, Shi C, et al. Embedding metric learning into setbased face recognition for video surveillance. Neuro Computing. 2015; 151(11): 1500-1506.

[13] De Giacomo, Lembo G, Lenzerini D, et al. Using ontologies for semantic data integration. In A comprehensive guide through the italian database research over the last 25 years. 2018: 87-202.

[14] Hughes R. Research agenda for the application of visualization to transportation systems. Journal of the Transportation Research Board. 2005; 1937(18): 145-151.

[15] Tian B, Gong L, Wang Y, et al. A method for exploring implicit concept relat-edness in biomedical knowledge network. Bmc Bioinformatics. 2016; 17(3): 265.

[16] Johnson AEW, Pollard TJ, Shen L, et al. Freely accessible critical care database. Nature Scientific Data. 2016; 39(3): 16-35.

[17] Soulakis ND, Carson MB, Lee YJ, et al. Visualizing collaborative electronic health record usage for hospitalized patients with heart failure. Journal of the American Medical Informatics Association Jamia. 2015; 122(8): 299-311. 